\title{
Multiple Brain Cysts: An Unusual Form of Demyelinating Disease
}

\author{
Mario Habek, Ivan Adamec, Kamelija Žarković, David Ozretić, Vesna V. Brinar
}

Can. J. Neurol. Sci. 2011; 38: 774-776

The course of multiple sclerosis (MS) can range from benign to malignant variants. One of the biggest problems in MS diagnosis is when a patient with magnetic resonance imaging (MRI) scan suggestive of MS has no clinical symptoms. In such instances the diagnosis of radiologically isolated syndrome (RIS) is usually made. In the four published series of patients with RIS, about one third of them go on to develop clinically isolated syndrome (CIS) in a period of three years. ${ }^{1}$ In most of these patients, brain MRI was performed because of chronic daily headache.

We present a patient with multiple brain cyst who after extensive investigations proved to have an unusual form of RIS.

\section{Case Report}

A previously healthy, 22-year-old female student presented with a two month history of constant dull headaches. Her physical and neurological examinations were normal. A brain MRI was performed revealing multiple cystic post contrast rimenhancing lesions. Complete blood cell count (CBC) chemistry panel, coagulation tests, electrocardiogram (ECG) and chest Xray were normal. Laboratory tests including serum and cerebrospinal fluid (CSF) serology for Echinococcus, Cysticercus, Borrelia, syphilis, Toxoplasma, and serum serology for viruses (HBV, HCV and HIV) were all negative as well as tumor markers and paraneoplastic antibodies (alpha-fetoprotein, carcinoembryonic antigens, CA 125, CA 19.9, CA 15.3, Hu, Yo, $\mathrm{Ri}$ ). Immunological tests (antistreptolysin titre, rheumatoid factor, anti-DNA antibody, antinuclear antibody, antineutrophil cytoplasmic antibody, angiotensin-converting enzyme) and thyroid function tests were all within normal range. The CSF examination showed normal cell count and protein level, with positive oligoclonal IgG bands. Computed tomography (CT) of

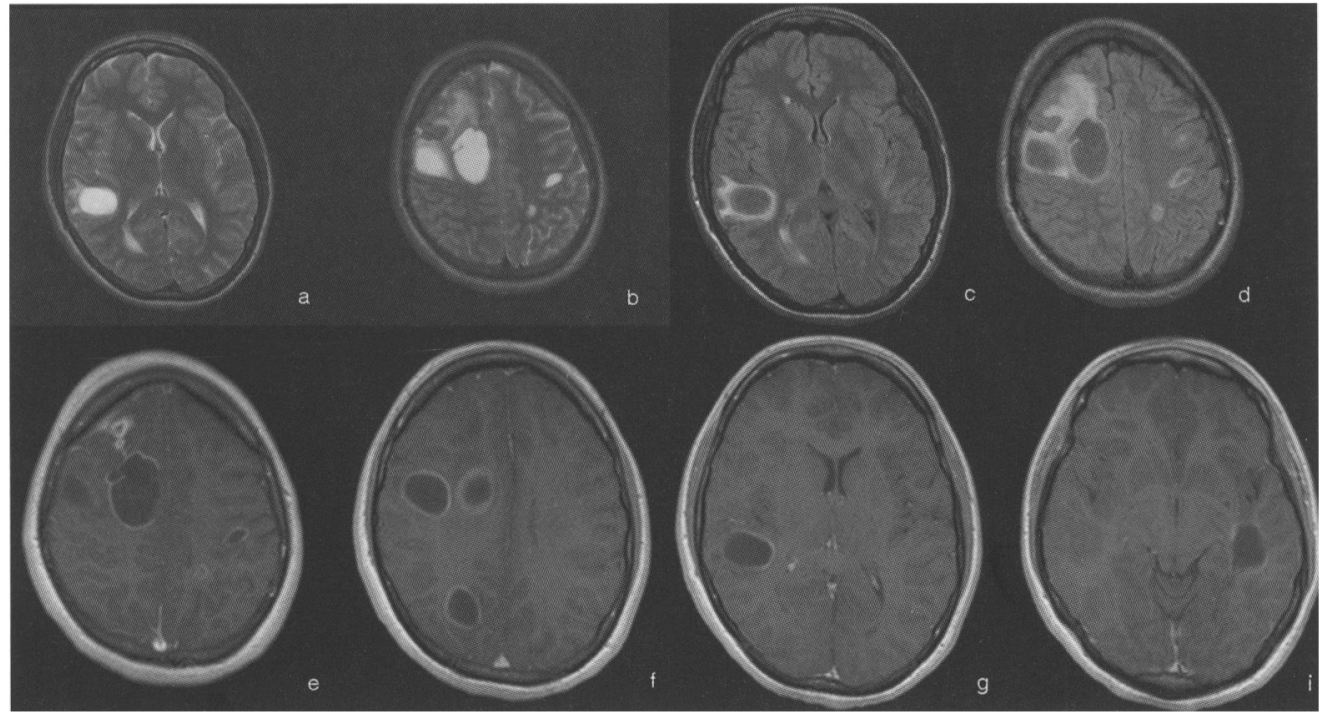

Figure 1: Brain MRI performed after the first biopsy, a) and b) T2-wighted images; $c$ ) and d) FLAIR images; e), f), g) and i) T1-weighted sequences following gadolinium application. Multiple cystic lesions, located cortico-subcorticaly, the biggest lesion is located in the right frontal cortex ( $37 \mathrm{~mm}$ ). After gadolinium administration ring enhancement of all lesions is visible. On DWI sequences there was no difusion restriction in the lesions. MR perfusion did not show neoangiogenesis within the lesion. Surrounding parenchima showed T2 and FLAIR hyperintensity - cytotoxic edema. Note the enhancement along bioptic pathway $(e)$.

From the Department of Neurology, Referral Center for Demyelinating Diseases of the Central Nervous System (MH, IA), Department of Pathology (KZ), Department of Radiology (DO), University Hospital Center Zagreb; School of Medicine (MH, KZ, DO, VVB), University of Zagreb, Zagreb, Croatia.

Received February 22, 2011. Final Revisions SubmitTed April 28, 2011.

Correspondence to: Mario Habek, University Department of Neurology, Zagreb School of Medicine and University Hospital Center, Kišpatićeva 12, HR-10000, Zagreb, Croatia. 


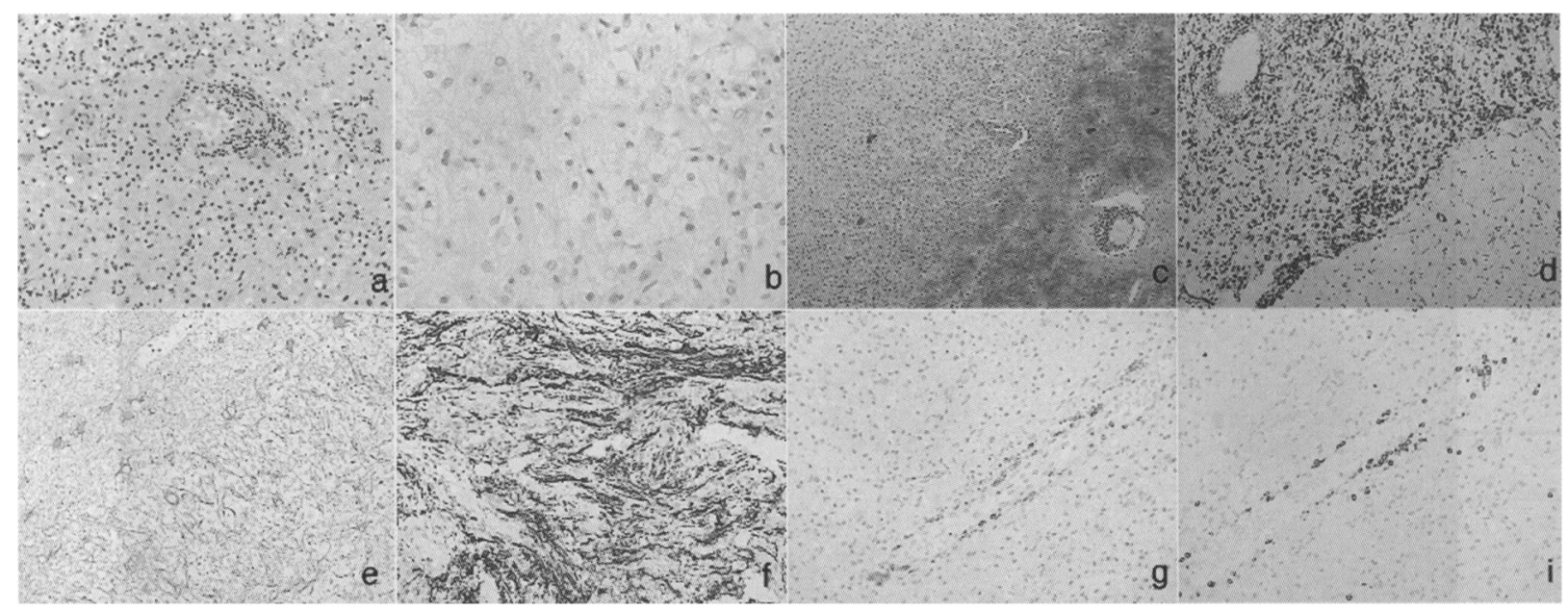

Figure 2: Hematoxylin and eosin $(H \& E)$ staining showed extensive inflammation with profound perivascular inflammatory infiltrates and diffuse dispersion of inflammation into the parenchyma ( $a$ and $b$ ). In addition there were focal lesions with extensive macrophage infiltration, protoplasmic gliosis, and in some areas, severe tissue distruction and formation of small cysic cavities. Staining for myelin (Klluver-Barrera) revealed profound perivenous demyelination in the white matter as well as the formation of large confluent plaques of demyelination (c). Immunocytochemistry for CD68 revealed densely packed macrophages within the lesions and profound microglia activation in the surrounding normal appearing grey and white matter (d). Profound protoplasmic astrocytic gliosis became evident in sections stained for GFAP (e). Staining for axons showed clear evidence of primary demyelination with partial preservation of axons $(f)$. Aquaporin 4 immunoreactivity was reduced within the lesions, but a specific loss of aquaporin 4 , as present in fresh lesions of NMO was absent. CD2O positive $B$ cells were rare and mainly restricted to the perivascular space ( $(\mathrm{g})$. The inflammatory infiltrates contained numerous $C D 3$ positive T-cells $(h)$ in the perivascular infiltrates and diffusely dispersed within the lesion parenchyma.

the abdomen and pelvis were normal as well as echocardiogram and breast and gynecologic ultrasound.

A stereotactic biopsy of the largest cyst in the right frontal lobe was performed. (Figure 1.) Pathohistological analysis revealed areas of demyelinating inflammation which were strictly limited from the surrounding brain tissue. Because pathological report was inconclusive, a month later an open brain biopsy was performed. (Figure 2). The pathological features of this lesion were consistent with a multiple sclerosis like acute inflammatory demyelinating disease with unusually destructive lesions. A neuromyelitis optica spectrum disorder was unlikely, since there was no astrocytic complement staining and no selective astrocyte and aquaporin 4 loss. On pathological grounds, it is most likely that this case represents fulminate multiple sclerosis.

The patient was started on intravenous corticosteroid therapy (methylprednisolone $1000 \mathrm{mg}$ for five days). One week later a brain MRI was performed showing reduction in the size of the lesion in the right frontal lobe with postcontrast enhancement of the dura on the place of the biopsy and posterior parts of the lesion. All other lesions showed reduction in size, and only lesion in the left frontal lobe subocrticaly showed very mild enhancement. (Figure 3).

She was released from the hospital in good health and symptom free.

\section{Discussion}

Presentation of multiple sclerosis with cystic brain lesions is rare and has been reported only in few case reports..$^{2-4} \mathrm{Kim}$ and colleagues reported an 18-year-old patient with severe neurological deficit requiring plasma exchange, with MRI finding of multiple cystic lesions with incomplete ring enhancement. ${ }^{2}$ These authors have suggested that the appearance of cystic lesions with open ring enhancement and the lack of mass effect or surrounding edema, despite the large size of the lesions, may be of value in differentiating demyelinating from nondemyelinating cysts. The second report is of a 14-year-old boy with already established diagnosis of MS, who developed new neurological symptoms. ${ }^{3}$ His brain MRI showed two cystic ring enhancing lesions, however brain biopsy was inconclusive, showing just reactive gliosis. Another two cases of cystic appearance of MS lesions emphasized the fact that the correlation between clinical status and MRI findings in MS patients is weak. ${ }^{4}$ Large cystic lesions in MS usually do not cause symptoms related to the mass effect. Follow-up of such patients is important because, in cases of MS, the clinical course (developing of new symptoms) and MRI appearance (shrinkage of lesions and appearance of new lesions, usually more consistent with MS) will change, so some cerebral biopsies can be avoided.

Multiple sclerosis is not the only idiopathic inflammatory demyelinating disease that can form cysts. They are relatively common in other demyelinating diseases, such as neuromyelitis optica (NMO) and acute disseminated encephalomyelitis (ADEM). In NMO, the necrosis and cavitations affect the grey matter and the white matter in spinal cord and optic nerves. In ADEM, on the other hand, signal abnormalities, in contrast to MS or NMO, usually resolve markedly, along with clinical improvement following high dose steroid therapy. And, although multiple cystic lesions and large focal tumor like cysts have been reported at onset of ADEM, rarely they can be a sequel of this disease. The diagnosis of ADEM or NMO was highly unlikely in our patient. She did not experience any clinical symptoms beside 


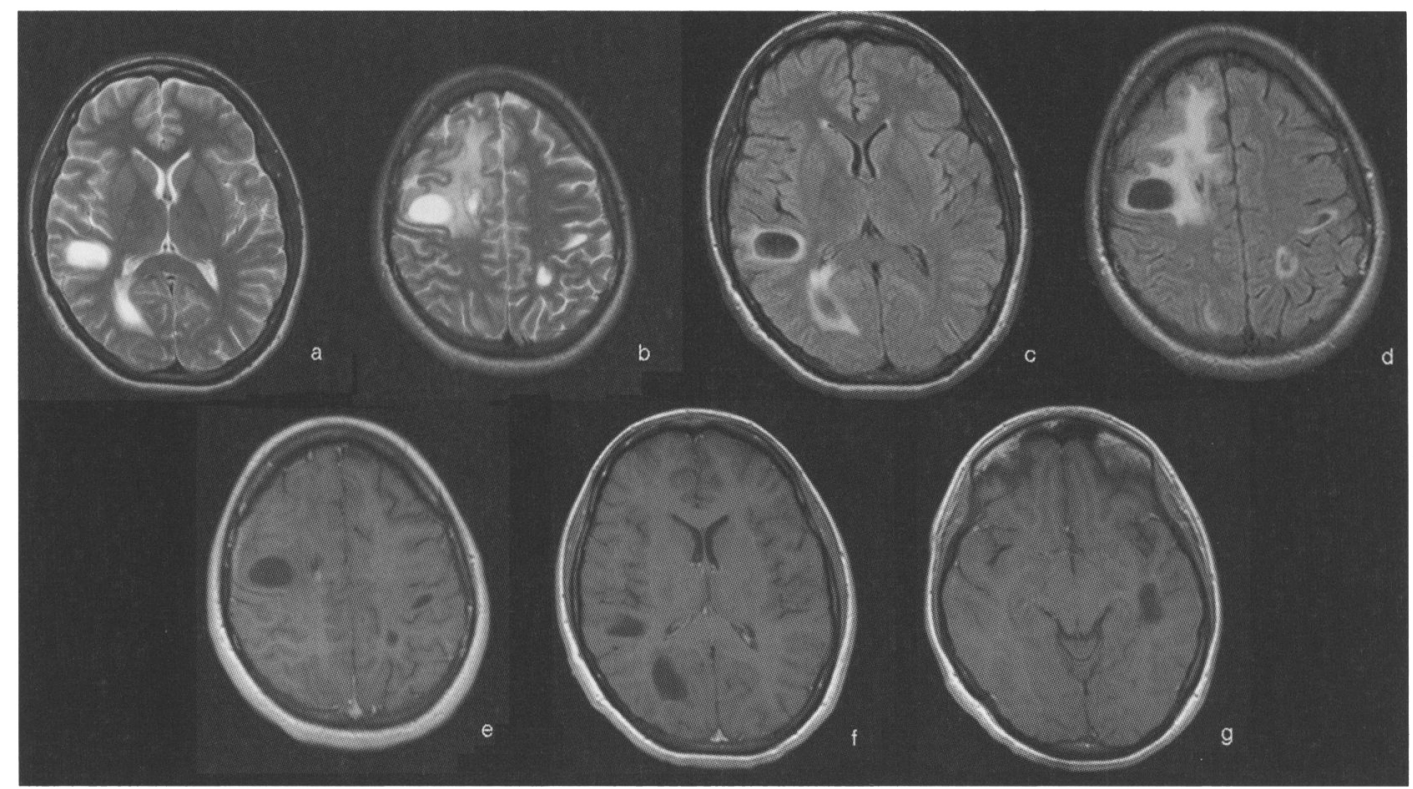

Figure 3: Brain MRI performed one week after treatment with steroids, a) and b) T2-wighted images; c) and d) FLAIR images; e),f) and g) TI-weighted sequences following gadolinium application. Right osteoclastic craniotomy is seen with mild postoperative edema of the right frontal lobe. The number of lesions is the same comparing to Figure 1, however the lesions are smaller, and only the lesion that was biopsed showed postcontrast enhancement.

headache, which is not a feature of neither of these diseases. However due to relatively limited follow up (six months) and in absence of confirmation of new disease activity (clinically or by MRI) an unusual case of ADEM can not be excluded. More compelling for Relapsing/Remitting Multiple Sclerosis (RRMS) would be follow up with evidence of recurrent disease activity. While the existence of subtle and even 'clinically silent' presentations that are eventually ascertained as MS are now appreciated, it remains possible that some ADEM presentations could be subtle as well.

One of the unresolved questions is whether to treat patients with incidental findings on brain MRI, without clinical symptoms. The rationale to treat with immunomodulatory drugs lies in few studies showing that RIS patients present similar features to MS patients. ${ }^{2}$ Corticosteroids are mainstream initial treatment of demyelinating diseases, with plasma exchange as an option when steroids do not work..$^{5}$ We decided to treat our patient with steroids due to the severe destructive nature of the lesions and intractable headaches.

This case study demonstrated a patient with large multiple cystic lesions in the brain which showed no mass effect. Seewann et al. reviewed reported findings of atypical MRI characteristics of idiopathic inflammatory demyelinating lesions of the brain and classified them into four different subtypes: "megacystic", "ring-like", "Balo-like" and "infiltrative". The megacystic subtype was characterized by large $(\geq 3 \mathrm{~cm}$ in diameter) cyst-like lesions which showed rim of contrast enhancement and were mostly solitary. ${ }^{6}$ The MRI of our patient showed multiple cystic lesions with the diameter of the largest one of $3.7 \mathrm{~cm}$ and rim of contrast enhancement on T1-weighted images which would place it in the megacystic subtype.
A morphological classification of atypical demyelinating lesions, such as mentioned above, would lead to inclusion of demyelination in the differential diagnosis of multiple cystic brain lesions and aid in correctly diagnosing such patients.

\section{AUTHORS' CONTRIBUtions}

Study concept and design: Habek, Adamec, Brinar. Acquisition of data: Habek, Adamec, Žarković, Ozretić, Brinar. Analysis and interpretation of data: Habek, Adamec, Žarković, Ozretić, Brinar. Drafting of the manuscript: Habek, Adamec. Critical revision of the manuscript for important intellectual content: Habek, Adamec, Žarković, Ozretić, Brinar. Administrative, technical, and material support: Habek, Adamec, Žarković, Ozretić.

\section{REFERENCES}

1. Chataway J. When confronted by a patient with the radiologically isolated syndrome. Pract Neurol. 2010;10:271-7.

2. Kim $\mathrm{P}, \mathrm{McDougall} \mathrm{A}$. Acute demyelinating disorder appearing as multiple cystic brain lesions. Arch Neurol. 2004;61:966-7.

3. Chakrabortty S, Nagashima T, Saitoh M, Hanada Y, Hiyama K, Tamaki N. Intracerebral ring-enhancing lesions in a patient with multiple sclerosis: a case report. Surg Neurol. 1995;43:591-4.

4. Kilincoglu BF, Altinors N, Caner H, Cekinmez M, Albayrak AH, Bavbek M. Pseudotumor cystic demyelinating plaque: report of two cases. Turk Neurosurg. 2003;13:61-6.

5. Habek M, Barun B, Puretic Z, Brinar VV. Treatment of steroid unresponsive relapse with plasma exchange in aggressive multiple sclerosis. Ther Apher Dial. 2010;14:298-302.

6. Seewan A, Enzinger C, Filippi M, et al. MRI characteristics of atypical idiopathic inflammatory demyelinating lesions of the brain. J Neurol. 2008;255:1-10. 